\title{
Markov Chain Monte-Carlo Models of Starburst Clusters
}

\author{
Jorge Melnick \\ European Southern Observatory \\ email: jmelnick@eso.org
}

\begin{abstract}
There are a number of stochastic effects that must be considered when comparing models to observations of starburst clusters: the IMF is never fully populated; the stars can never be strictly coeval; stars rotate and their photometric properties depend on orientation; a significant fraction of massive stars are in interacting binaries; and the extinction varies from star to star. The probability distributions of each of these effects are not a priori known, but must be extracted from the observations. Markov Chain Monte-Carlo methods appear to provide the best statistical approach. Here I present an example of stochastic age effects upon the upper mass limit of the IMF of the Arches cluster as derived from near-IR photometry.
\end{abstract}

Keywords. Methods: statistics; Stars: evolution, atmospheres, rotation.

\section{Introduction}

The IMF is considered to be fundamental to understand massive star formation (although we have argued elsewhere that the IMF is immanent in the fractal structure of the ISM; eg. Melnick 2009). Starburst clusters are the best places where the IMF can be measured: they contain large numbers of massive stars - important to constrain stochastic effects, and their youth allows to control evolutionary effects. Yet, however young, the stellar populations cannot be strictly coeval, and, I will argue in this paper, even small age differences among the most massive stars have significant effects on the IMF.

A particularly interesting issue that has emerged recently is whether there is an upper limit to how massive stars can be. Crowther et al. (2010) found that the most massive stars in 30 Doradus and NGC3603 may have initial masses well above the hitherto canonical mass limit of $120 M_{\odot}$. In our photometric study of the Arches cluster (Espinoza et al. 2009), we had difficulties matching the most luminous stars to the most massive Geneva models, which left us with the distinct impression that some of these stars could be substantially more massive than $120 M_{\odot}$. So I endeavoured to reanalyse our Arches photometry using the most recent Geneva tracks, in particular those including rotation.

Raphael Hirschi kindly provided me with his tracks for masses up to $500 M_{\odot}$ (used in Crowther et al. 2010), but, although the potential impact of extended atmospheres on the photometric properties of massive stars is well known, there are as yet no comprehensive libraries of such models to be used for population studies. So to compute synthetic colours I used (Castelli \& Kurucz 2003) atmospheres. Here I present very preliminary results of comparing this new grid of synthetic photometry with the observations of the Arches cluster.

\section{The Arches Starburst cluster}

The left panel of Figure 1 shows the ZAMS for the Geneva tracks without rotation and the evolutionary track of a $120 M_{\odot}$ star, compared to the best photometric data for 

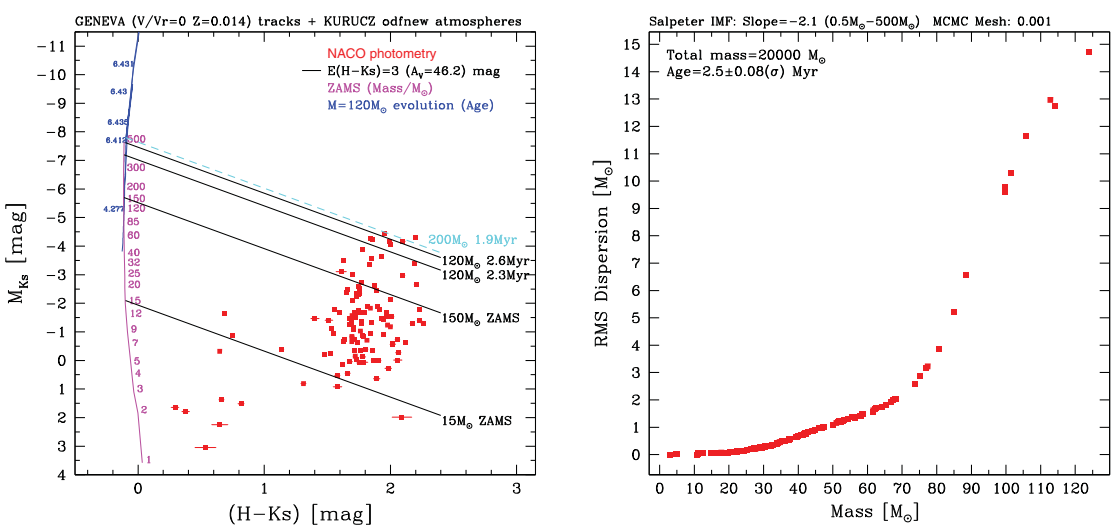

Figure 1. Left. NACO adaptive-optics photometric observations of Arches compared to evolutionary tracks. The ages in the $120 M_{\odot}$ track are labelled. The reddening vectors for stars of different masses and ages are shown as solid lines. The dashed line shows the reddening vector for a 1.9 Myr old star of $200 M_{\odot}$. Right. Rms dispersion versus mass for $10^{5}$ Monte-Carlo draws from a Gaussian age distribution of mean age 2.5 Myr and dispersion 0.08 Myr.

the stars in the Arches cluster from Espinoza et al. (2009). This figure illustrates how the inferred initial masses of the most luminous stars depend critically on age: at $2.6 \mathrm{Myr}$ the masses would not be significantly larger than $120 M_{\odot}$ whereas at $2.3 \mathrm{Myr}$, the most luminous stars would be more massive than $200 M_{\odot}$.

By definition, starburst clusters have ages comparable to the main-sequence life times of their most massive stars: all the massive stars form in a short burst. Barring an act of god, however, this burst cannot be infinitely short, and specifically, cannot be shorter than the free-fall time $t_{F F}$ of parent cloud. For a Virialized cluster $t_{\mathrm{FF}}$ is given by $t_{\mathrm{FF}}=1 / \sqrt{G \rho} \simeq 0.0243 \times M_{\mathrm{cl}} / \sigma_{\mathrm{cl}}^{3}$ Myr where $\sigma_{\mathrm{cl}}=0.0927 \times \sqrt{M_{\mathrm{cl}} / R_{\mathrm{cl}}} \mathrm{km} \mathrm{s}^{-1}$.

\subsection{Results and Future Prospects}

There is some debate about the age of the Arches cluster, but since I am using the same photometric data, here I will use the values of Espinoza et al. (2009): age $=2.5 \mathrm{Myr}$; mass $M_{\mathrm{cl}}=2 \times 10^{4} M_{\odot}$; radius $R_{\mathrm{cl}}=0.5 \mathrm{pc}$, from which $t_{\mathrm{FF}} \sim 0.08 \mathrm{Myr}$. The right-hand panel of Figure 1 shows Monte-Carlo simulations for a Gaussian distribution of stellar ages with a mean of $2.5 \mathrm{Myr}$ and a dispersion of $0.08 \mathrm{Myr}$. The average upper mass limit of the IMF is $\sim 125 M_{\odot}$ with rms dispersion of $15 M_{\odot}$. There is no strong evidence in the photometry, therefore, that the Arches cluster may host stars more massive than $140 M_{\odot}$.

The legacy ground-based and HST data sets of 30Dor that are becoming available will allow to use MCMC methods to derive the parent distributions of all the stochastic parameters that define the stellar populations of this iconic starburst cluster: age; extinction; rotation; and binarity. This contribution marks the beginning of such program.

\section{References}

Castelli, F. \& Kurucz, R. L. 2003, in N. Piskunov, W. W. Weiss, \& D. F. Gray (eds.), Modelling of Stellar Atmospheres, Vol. 210 of IAU Symposium, p. 20P

Crowther, P. A., Schnurr, O., Hirschi, R., et al. 2010, MNRAS 408, 731

Espinoza, P., Selman, F. J., \& Melnick, J. 2009, A\& $A$ 501, 563

Melnick, J. 2009, in Revista Mexicana de Astronomia y Astrofisica Conference Series, Vol. 37 of Revista Mexicana de Astronomia y Astrofisica Conference Series, pp 21-31 